\title{
Análise comparativa da qualidade de vida de idosas praticantes de exercícios físicos em centros esportivos e nas academias da terceira idade
}

Jaqueline Fernandes da Silva*, José Roberto Andrade do Nascimento Júnior**, Ana Paula Serra de Araújo***, Daniel Vicentini de Oliveira****

\section{Resumo}

O presente estudo teve como objetivo analisar a qualidade de vida de idosas praticantes de exercícios físicos em dois locais distintos, nos centros esportivos e nas Academias da terceira idade (ATIs) do município de Maringá-PR, buscando especificamente comparar a qualidade de vida das idosas nesses locais. Foram avaliadas 70 idosas, sendo 35 praticantes de exercícios físicos nos centros esportivos (G1) e 35 nas ATIs (G2). A qualidade de vida foi avaliada por meio do WHOQOL-Bref e WHOQOL-Old. As idosas do G1 atingiram escore de 82,00 no WHOQOL-Bref, e o G2 77,67 ( $p=0,04)$. Já no WHOQOL-Old, o G1 apresentou escore de 103 e o G2 de 94 ( $p=0,01)$, indicando que as idosas praticantes de exercícios físicos em centros esportivos têm maior percepção de qualidade de vida se comparadas às idosas praticantes nas ATIs.

Palavras chave: Envelhecimento. Atividade física. Promoção da saúde.

\section{Introdução}

O envelhecimento, de um modo geral, é caracterizado por um conjunto de alterações estruturais e funcionais desfavoráveis do organismo que se adquire com avanço da idade. Essas modificações prejudicam o desempenho de habilidades motoras, dificultando a adaptação do indivíduo ao meio ambiente, desencadeando modificações não só de ordem física, mas, também, de ordem psicológica, social e econômica (CUNHA et al., 2009), que refletem na qualidade de vida dessa população (FREITAS et al., 2016).

A prática regular de exercícios físicos está associada à melhora da qualidade de vida (LIMA et al., 2016). Sabe-se que a

* Graduada em Educação Física pela Faculdade Metropolitana de Maringá (Famma). Email: jaquefernandes21@hotmail.com

** Graduado em Educação Física Doutor em Educação física (UEM). Professor Adjunto no Departamento de Educação física da Universidade Estadual do Vale do São Francisco (Unifasv). Email: jroberto.jrs01@gmail.com

**** Fisioterapeuta. Mestre em Promoção da Saúde pelo Centro Universitário Cesumar (Unicesumar). Email: anasaraujo@hotmail.com

**** Graduado em Educação Física e Fisioterapia pelo Centro Universitário de Maringá; Mestre em Promoção da Saúde (Unicesumar); Doutorando em Gerontologia pela Universidade Estadual de Campinas (Unicamp); Professor Adjunto do Departamento de Educação física da Faculdade Metropolitana de Maringá (Famma). Email: d.vicentini@hotmail.com Endereço para correspondência: Avenida Londrina, 934, apto 1907, torre A. CEP 87050-730, Maringá, PR.

$\rightarrow$ http://dx.doi.org/10.5335/rbceh.v13i2.5726

Recebido em: 12.02.2016. Aceito em: 17.10.2016. 
prática de exercício físico é fundamental para o bem-estar e a qualidade de vida do indivíduo, independentemente da sua idade, prevenindo o aparecimento de diversas doenças crônicas, algumas alterações físicas e até mesmo psíquicas (ALENCAR et al., 2010), podendo ser realizada em ambientes particulares como academias de musculação e ginástica, escolas de natação e hidroginástica, clubes recreativos e/ou ambientes de caráter público como os centros esportivos municipais e as Academias da Terceira Idade (ATIs) ou academias da saúde.

Tanto nos ambientes particulares como nos públicos, a prática de exercícios físicos por idosos facilita a interação entre eles, melhorando a socialização, o que tende a inferir positivamente em um bom nível de qualidade de vida (OLIVEIRA; BERTOLINI; BENEDETI, 2012).

A prática regular de atividade física deve ser bem orientada, exigindo a presença de um profissional de educação física (FAUSTINO et al., 2015). O profissional de educação física é apto para atuar no planejamento, na avaliação e na orientação de programas de atividades físicas para grupo de idosos saudáveis, bem como os que apresentam fatores de riscos para as doenças crônicas não transmissíveis (SANTOS et al., 2016).

O profissional de educação física está presente nos centros esportivos, pois ele contribui para prescrição correta de exercício físico, visando à melhora $\mathrm{e}$ à manutenção da saúde (BOETTGE et al., 2015). Além disso, as experiências vivenciadas nos encontros dos centros esportivos possibilitam a troca de sabe- res e conhecimento, tanto para os idosos quanto para os profissionais de educação física (NUNES et al., 2015).

Alguns projetos das ATIs são compostos por estagiários de educação física, ou profissionais já formados para realizar atividades de orientação, avaliação e acompanhamentos dos exercícios nos aparelhos. Porém, é uma realidade não frequentemente vista em todos os locais que apresentam os aparelhos (PALÁCIOS; NARDI, 2007). Portanto, esse profissional tem uma relevante atuação social no processo educativo para adesão de um estilo de vida saudável, e quando necessário ele pode intervir positivamente nos parâmetros que influenciam na qualidade de vida dos idosos (ESTEVES et al., 2010). Os municípios do Brasil que oferecem as ATIs reafirmam o compromisso com a população de cumprir a Política Nacional de Promoção da Saúde, priorizando ações de cuidados coletivos na atenção básica (SÁ et al., 2016).

Nesse contexto, o presente estudo teve como objetivo analisar a qualidade de vida de idosas praticantes de exercícios físicos em dois locais distintos, nos centros esportivos e nas ATIs do município de Maringá (PR), buscando especificamente comparar a qualidade de vida das idosas nesses locais.

\section{Materiais e métodos}

Este estudo, de caráter descritivo e transversal, foi aprovado pelo Comitê de Ética do Centro Universitário Cesumar (Unicesumar), pelo protocolo número 1.157.110/2015.

RBCEH, Passo Fundo, v. I3, n. 3, p. 285-298, set./dez. 2016 
A população do estudo foi composta de idosas praticantes de exercícios em centros esportivos e ATIs do município de Maringá, estado do Paraná. Os centros esportivos escolhidos foram, segundo a Secretaria de Esportes e Lazer do município, aqueles com maior número de frequentadores, como o do Conjunto Mandacarú, Jardim Alvorada, Conjunto Três Lagoas e Vila Operária. As ATIs escolhidas, também, foram aquelas com maior fluxo como a localizada no Parque do Ingá, Avenida Mandacarú, Vila Operária e no Bairro Parigot.

Nos centros esportivos e ATIs selecionados para a realização da pesquisa, a amostra foi selecionada por conveniência e as voluntárias foram informadas quanto à justificativa, aos objetivos e procedimentos a serem realizados, conforme orientações para pesquisa com seres humanos constantes na Resolução 196/96 do Conselho Nacional de Saúde. Após esses procedimentos, as participantes assinaram o Termo de Consentimento Livre e Esclarecido (TCLE).

Foram incluídas no estudo idosas com idade igual ou superior a 60 anos, que praticam exercícios, exclusivamente, nos centros esportivos ou nas ATIs há pelo menos três meses e duas vezes semanais. Foram excluídos idosos do sexo masculino, praticantes de outras modalidades de exercício físico, e/ou com alterações neurológicas e psíquicas incapacitantes para a realização dos questionários. A amostra final foi composta de 70 idosas, sendo distribuídas em dois grupos: grupo de idosas praticantes de exercícios físicos nos centros esporti- vos (G1) cuja idade variou entre 62 e 70 anos (média de 66 anos), grupo de idosas praticantes de exercícios físicos nas ATIs (G2), cuja idade variou entre 66 e 76 anos (com média de 68 anos).

Para a aplicação desta pesquisa e caracterização das idosas, foi utilizado um questionário semiestruturado, contendo informações referentes à idade, ao estado civil, à situação ocupacional, à renda mensal em salário mínimo de referência no Censo Demográfico 2010 - IBGE, frequência semanal e tempo de prática de alongamento e ginástica. No item estado civil, serão consideradas solteiras também as idosas viúvas, separadas ou divorciadas.

A avaliação da qualidade de vida foi realizada por meio dos questionários WHOQOL-Bref e WHOQOL-Old. O WHOQOL-Bref é composto por 26 questões, das quais, duas referem-se à percepção individual da qualidade de vida e da condição de saúde, enquanto as demais, 24 questões, são subdivididas em quatro domínios: físico, psicológico, relações sociais e meio ambiente. Cada domínio pode alcançar escores de 4 a 20 , sendo que quanto mais próximo de 20 melhor a qualidade de vida do indivíduo no domínio avaliado. Somando-se os escores dos quatro domínios e das duas questões referentes à percepção do indivíduo, pode-se chegar a escores mínimos de 20 e máximo de 100. Quanto mais próximo de 100 , melhor a qualidade de vida global do avaliado (FLECK et al., 2000, CHACHAMOVICH; FLECK, 2008). 
O WHOQOL-Old consiste em 24 questões, atribuídas a seis facetas: funcionamento dos sentidos, autonomia, atividades passadas, presentes e futuras, participação social, morte e morrer e intimidade. Cada item é composto por quatro questões. Foi escolhido e utilizado o escore transformado, que é calculado a partir da conversão do escore bruto (apresentado a partir da somatória de cada questão, variando a pontuação entre 4 e 20) para uma escala de 0 a 100. A transformação de um escore bruto para um escore transformado da escala (ETE) entre 0 e 100 possibilita expressar o escore da escala em percentagem entre o valor mais baixo possível (0) e o mais alto possível (100). Para se obter o escore transformado da faceta (ETF) (0-100), foi aplicado a seguinte regra de transformação: $\mathrm{ETF}=6,25 \times(\mathrm{EBF}-4)$. Quanto mais próximo de 100, melhor a qualidade de vida no domínio avaliado (LEAL et al., 2009; PEDROSO; PILLATI; GUTIERREZ, 2010).

Para a análise dos dados, foi utilizado frequência e percentual para as variáveis categóricas. Para as variáveis numéricas, inicialmente, foi verificada a normalidade dos dados por meio do teste Kolmogorov-Smirnov. Como os dados não apresentaram distribuição normal, foram utilizadas Mediana (Md) e Quartis (Q1; Q3) para a caracterização dos resultados. Como se pretendia verificar as maiores percepções em relação aos domínios e facetas de qualidade de vida dos sujeitos, de uma forma geral, foi utilizado o ANOVA de Medidas Repetidas, após a verificação da esfericidade dos dados, seguido do Post Hoc de Bonferroni corrigido $(p<0,01)$. Na comparação entre os grupos (centros esportivos e ATIs), foi utilizado o teste "U" de Mann-Whitney, sendo adotada a significância de $\mathrm{p}<0,05$.

\section{Resultados}

Na Tabela 1, é possível observar o perfil sociodemográfico dos dois grupos, não influenciando nas análises seguintes. Conforme mostra a Tabela 1, a maioria das idosas do G1 era casada $(77,1 \%)$ e as do G2 eram solteiras (54,3\%). 
Tabela 1 - Distribuição de frequência do perfil das idosas praticantes de exercícios em centro esportivos e ATIs da cidade de Maringá-PR

\begin{tabular}{l|c|c|c|c}
\hline \multirow{2}{*}{ VARIÁVEIS } & \multicolumn{2}{|c|}{ Centro Esportivo } & \multicolumn{2}{|c}{ ATI } \\
\cline { 2 - 4 } & $\boldsymbol{f}$ & $\%$ & $\boldsymbol{f}$ & $\%$ \\
\hline Estado civil & 27 & 77,1 & 16 & 45,7 \\
$\quad$ Casada & 08 & 22,9 & 19 & 54,3 \\
$\quad$ Solteira & 03 & 8,6 & 05 & 14,3 \\
Tempo de Prática & 17 & 48,6 & 13 & 37,1 \\
3 meses a 1 ano & 15 & 42,9 & 17 & 48,6 \\
1 a 5 anos & & & & \\
$\quad$ Mais de 5 anos & 00 & 0,0 & 00 & 0,0 \\
Situação ocupacional & 35 & 100,0 & 35 & 100,0 \\
$\quad$ Ativo & & & & \\
$\quad$ Inativo & 03 & 8,6 & 05 & 14,3 \\
Renda mensal & 20 & 57,1 & 22 & 62,9 \\
$\quad$ Menos de 1 salário mínimo & 12 & 34,3 & 08 & 22,9 \\
1 a 2 salários mínimos & & & & \\
Mais de 2 salários mínimos & 11 & 31,4 & 06 & 17,1 \\
Escolaridade & 11 & 31,4 & 18 & 51,4 \\
$\quad$ Menos de 1 ano & 1 & 2,9 & 02 & 5,7 \\
1 a 4 anos & 12 & 34,3 & 09 & 25,7 \\
5 8 anos & & & & \\
$\quad$ Mais de 8 anos & 04 & 11,4 & 01 & 2,9 \\
Uso de Medicamentos & 31 & 88,6 & 34 & 97,1 \\
$\quad$ Menos de 3 & & & & \\
3 ou mais &
\end{tabular}

Fonte: primária.

Nota: f:frequência.

Ainda de acordo com a Tabela 1, observou-se que as idosas do G1, em sua maioria $(48,6 \%)$ praticavam exercícios nos centros esportivos de 1 a 5 anos e, no G2, o mesmo percentual de idosas praticavam exercícios nas ATIs há mais de cinco anos. Ambos os grupos apresentaram todas as idosas com situação ocupacional inativa, ou seja, não trabalham para renda própria. Verificou-se também que a maioria das idosas do G1 e G2 tinha renda mensal de um a dois salários mínimos apenas (57,1\% e 62,9\%, respectivamente). Em ambos os grupos, a maioria (G1-88,6\% e G2-97,1\%) utiliza mais de três medicamentos
A avaliação da qualidade de vida foi realizada por meio dos questionários WHOQOL Bref e WHOQOL Old, os quais apresentam escore máximo de 100 e 120 , respectivamente. Quanto mais próximo do escore máximo, melhor a avaliação da qualidade da idosa pesquisada.

A Tabela 2 apresenta a comparação dos domínios de avaliação da qualidade predominante nas mulheres praticantes de exercício, sem dividi-las entre grupos, a fim de verificar os domínios e as facetas predominantes na amostra de uma forma geral. 
Tabela 2 - Comparação dos domínios da avaliação da qualidade (WHOQOL Brefe Old) predominantes nas idosas praticantes de exercício

\begin{tabular}{l|c}
\hline \multicolumn{1}{c}{ VARIÁVEIS } & Md (Q1; Q3) \\
\hline \multicolumn{2}{c}{ Domínios de Qualidade de Vida (WHOQOL Bref) } \\
\hline Domínio 1 - Físico & $16,67(14,67 ; 18,00)^{\mathrm{a}}$ \\
Domínio 2 - Psicológico & $16,00(16,00 ; 17,33)^{\mathrm{b}}$ \\
Domínio 3 - Relações sociais & $16,00(16,00 ; 20,00)$ \\
Domínio 4 - Meio ambiente & $13,00(12,00 ; 14,00)^{\mathrm{c}}$ \\
Domínio 5 - Autoavaliação & $16,00(16,00 ; 20,00)$ \\
\hline Facetas de Qualidade de Vida (WHOQOL Old) \\
\hline Faceta 1 - Funcionamento dos Sentidos & $16,00(16,00 ; 19,00)^{\mathrm{d}}$ \\
Faceta 2 - Autonomia & $16,00(16,00 ; 19,00)$ \\
Faceta 3 - Atividades & $16,00(16,00 ; 17,00)$ \\
Faceta 4 -Participação Social & $16,00(16,00 ; 16,00)^{\mathrm{e}}$ \\
Faceta 5 - Morte e Morrer & $20,00(16,00 ; 20,00)$ \\
Faceta 6 - Intimidade & $20,00(4,00 ; 20,00)$ \\
\hline Fonte: primária. & \\
Nota: * Diferença significativa (ANOVA de Medidas - p < 0,01): p < 0,01 entre: a) Dom 1 e Dom 3, 4 e 5; b) Dom 2 e Dom 4 \\
$\quad$ e 5; c) Dom 4 e Dom 3 e 5; d) Fac 1 e Fac 4; e) Fac 4 e Fac 5.
\end{tabular}

Conforme se depreende da Tabela 2, os domínios de avaliação da qualidade do WHOQOL Bref que mais prevaleceram entre as idosas foram o domínio Físico $(\mathrm{Md}=16,67)$ e Autoavaliação ( $\mathrm{Md}=$ 16,00 ), visto que apresentou diferença significativa $(\mathrm{p}<0,01)$ com o domínio que apresentou menor valor, o Meio Ambiente $(\mathrm{Md}=13,00)$. Em relação ao WHOQOL Old, as facetas com maiores valores foram Morte e Morrer $(\mathrm{Md}=$ 20,00) e Funcionamento dos Sentidos $(\mathrm{Md}=16,00)$, pois apresentaram diferen- ça significativa $(\mathrm{p}<0,01)$ com o domínio que apresentou menor valor, o de Participação Social $(\mathrm{Md}=16,00)$.

Conforme a Tabela 3, na avaliação pelo WHOQOL-Bref, as idosas do G1 possuem maior qualidade de vida $(82,00)$ do que as do G2 $(77,67)$, com resultado significativo $(\mathrm{p}=0,04)$. Na avaliação pelo WHOQOL-Old, as idosas do G1 possuem também avaliação da qualidade de vida maior $(103,00)$ do que as do G2 $(94,00)$ $(p=0,01)$.

Tabela 3 - Comparação da avaliação da qualidade total (WHOQOL Bref e Old) das idosas do G1 e do G2

\begin{tabular}{|c|c|c|c|}
\hline \multirow{2}{*}{ VARIÁVEIS } & G1 $(n=35)$ & G2 $(n=35)$ & \multirow{2}{*}{ p-valor } \\
\hline & Md (Q1; Q3) & Md (Q1; Q3) & \\
\hline $\begin{array}{l}\text { WHOQOL-Bref } \\
\text { Qualidade Total }\end{array}$ & $82,00(77,16 ; 88,17)$ & $77,67(74,66 ; 84,17)$ & $0,04^{*}$ \\
\hline $\begin{array}{l}\text { WHOQOL-OId } \\
\text { Qualidade Total }\end{array}$ & $103,00(99,00 ; 108,00)$ & $94,00(88,00 ; 104,00)$ & $0,01^{*}$ \\
\hline
\end{tabular}

Fonte: primária.

Nota: * Diferença significativa entre G1 e G2: p < 0,05 - Teste "U” de Mann-Whitney.

G1: praticante de exercícios físicos nos centros esportivos; G2: praticante de exercícios nas ATIs. 
A Tabela 4 apresenta a comparação dos domínios e facetas de avaliação da qualidade das idosas do G1 e G2.

Tabela 4 - Comparação da flexibilidade e dos domínios e facetas de avaliação da qualidade das idosas do G1 e G2

\begin{tabular}{|c|c|c|c|}
\hline \multirow[t]{2}{*}{ VARIÁVEIS } & $\begin{array}{c}\text { G1 } \\
(n=35)\end{array}$ & $\begin{array}{ll}\text { G2 } & \text { (n } \\
& =35)\end{array}$ & \multirow[t]{2}{*}{$P$} \\
\hline & Md $(Q 1 ; Q 3)$ & Md $(Q 1 ; Q 3)$ & \\
\hline \multicolumn{4}{|c|}{ Domínios de Qualidade de vida (WHOQOL-Bref) } \\
\hline Domínio 1 - Físico & $17,33(16,00 ; 18,00)$ & $16,00(14,67 ; 17,33)$ & 0,06 \\
\hline Domínio 2 - Psicológico & $16,00(16,00 ; 17,33)$ & $16,00(15,33 ; 16,67)$ & 0,26 \\
\hline Domínio 3 - Relações sociais & $16,00(16,00 ; 20,00)$ & $16,00(16,00 ; 16,00)$ & $0,02^{*}$ \\
\hline Domínio 4 - Meio ambiente & $12,50(12,00 ; 13,50)$ & $13,50(12,00 ; 14,50)$ & 0,38 \\
\hline Domínio 5 - Autoavaliação & $20,00(16,00 ; 20,00)$ & $16,00(16,00 ; 18,00)$ & $0,01^{*}$ \\
\hline \multicolumn{4}{|c|}{ Facetas de Qualidade de vida (WHOQOL-OId) } \\
\hline Faceta 1 - Func. dos Sentidos & $17,00(16,00 ; 19,00)$ & $16,00(16,00 ; 19,00)$ & 0,08 \\
\hline Faceta 2 - Autonomia & $16,00(16,00 ; 17,00)$ & $16,00(16,00 ; 19,00)$ & 0,88 \\
\hline Faceta 3 - Atividades & $16,00(16,00 ; 16,00)$ & $16,00(16,00 ; 17,00)$ & 0,31 \\
\hline Faceta 4 -Participação Pessoal & $16,00(16,00 ; 16,00)$ & $16,00(16,00 ; 16,00)$ & 0,87 \\
\hline Faceta 5 - Morte e Morrer & $20,00(16,00 ; 20,00)$ & $20,00(16,00 ; 20,00)$ & 0,89 \\
\hline Faceta 6 - Intimidade & $20,00(20,00 ; 20,00)$ & $10,00(4,00 ; 20,00)$ & $0,01^{*}$ \\
\hline
\end{tabular}

Fonte: primária.

Nota: * Diferença significativa entre os G1 e G2: $\mathrm{p}<0,05$ - Teste "U” de Mann-Whitney.

G1: praticante de exercícios físicos nos centros esportivos; G2: praticante de exercícios nas ATIs.

Em relação aos domínios do WHOQOL-Bref, houve diferença significativa nas Relações sociais $(\mathrm{p}=0,02)$ e na autoavaliação da qualidade de vida ( $\mathrm{p}=0,01)$, evidenciando melhor percepção para as idosas do G1. Quanto às facetas do WHOQOL-Old, houve diferença significativa apenas na faceta de Intimidade $(p=0,01)$, sendo melhor no G1.

\section{Discussão}

Este estudo teve como objetivo analisar a qualidade de vida de idosas usuárias dos centros esportivos e das ATIs do município de Maringá-PR, buscando comparar a essas variáveis entre os grupos.
Primeiramente, sem separação dos grupos, a qualidade de vida no domínio Físico (do WHOQOL-Bref) apresentou o melhor escore $(\mathrm{Md}=16,67)$, ou seja, o exercício físico supostamente está evitando dores, consequentemente, diminuindo idas ao médico, dando energia para essas idosas, fazendo com que elas aceitem sua aparência física, melhorando seu sono, suas atividades de vida diária e sua capacidade para o trabalho, visto que são estas as questões referentes ao domínio Físico, no questionário WHOQOL-Bref. $\mathrm{O}$ exercício físico regular promove um efeito benéfico sobre a qualidade de vida da população longeva (SANCHEZ; BRASIL; FERREIRA, 2014). 
Outro domínio que apresentou dados positivos foi o de Autoavaliação $(\mathrm{Md}=16,00)$, ou seja, ao serem questionadas como avaliam sua qualidade de vida e quão satisfeitas estão com sua saúde, as respostas foram positivas, portanto a prática de exercícios para essas idosas possivelmente esteja auxiliando na melhora da saúde e, consequentemente, na avaliação da qualidade de vida.

Em um estudo realizado por Andrade e Martins (2011) avaliaram a qualidade de vida e analisaram a influência dos fatores sociodemográficos nos idosos por meio de um estudo transversal. Observaram que quanto menor é a idade, mais elevada é a qualidade de vida e funcionalidade familiar nos idosos.

Em relação ao WHOQOL Old, as facetas com maiores valores foram Morte e Morrer e Funcionamento dos Sentidos, pois apresentaram diferença significativa $(\mathrm{p}<0,01)$ com o domínio que apresentou menor valor, o de Participação Social $(\mathrm{Md}=16,00)$.

As facetas Morte e Morrer $(\mathrm{Md}=$ 20,00) e Funcionamento dos Sentidos $(\mathrm{Md}=16,00)$ apresentaram resultados positivos e significativos $(\mathrm{p}<0,01)$ em relação a menor faceta, Participação social $(M d=16,00)$. Em relação à faceta Morte e Morrer, a prática de exercício físico pode estar auxiliando na diminuição de momentos de medo e de preocupação acerca de se irão ou não sofrer, nem mesmo com a maneira que irão morrer.

No estudo de Padoin et al. (2010) ao avaliar o risco de quedas em idosos sedentários e os que praticam atividade física, obteve como resultado que ativos apresentavam melhores desempenhos no equilíbrio na marcha, já os sedentários apresentaram maiores probabilidades do risco de quedas.

Ao avaliar a capacidade física e funcional e a qualidade de vida dos idosos portadores de doenças crônicas, Ozturk et al. (2011) realizaram um estudo com 100 idosas e pôde-se verificar que existem diferenças entre os sexos em relação à dor, à quantidade e ao tipo de doenças crônicas, ao nível de mobilidade, ao estado funcional e à qualidade de vida. Assim, programas de reabilitação para melhorar a capacidade física e funcional em idosos e programas e ações que aumentam a participação em atividades são necessários para reduzir a dor e melhorar a qualidade de vida.

Os benefícios que a prática de atividade física sistematizada proporciona estão relacionados com a manutenção da saúde, independência e autonomia dos idosos (MOREIRA; TEIXEIRA; NOVAES, 2014).

Conforme a Tabela 4, na avaliação pelo WHOQOL-Bref e Old, as idosas do G1 possuem melhor qualidade de vida do que as do grupo G2 com diferença estatisticamente significativa entre os grupos. Essa diferença significante na qualidade e vida entre os grupos, nos dois questionários, pode ser explicada pelo fato de que os exercícios desenvolvidos nos centros esportivos são realizados por meio da supervisão de profissionais qualificados, o que promove maior segurança e motivação, gerando, assim, possivelmente, uma maior eficiência e, consequentemente, avaliação da qua- 
lidade. Ou seja, as aulas nos centros esportivos são estruturadas, montadas e em horários específicos, tendo praticamente a presença das mesmas idosas, o que facilita a interação entre elas e o grupo, melhorando a socialização, que faz parte de uma boa avaliação da qualidade, o que segundo Oliveira, Bertolini e Benedeti (2012) promovem maior socialização, modificando a ideia de que, com o envelhecimento, vem o isolamento social e a solidão.

Além disso, os centros esportivos oferecem uma maior variedade de modalidades estruturadas, com aquecimento, parte principal e volta à calma, como ginástica localizada, ginástica voltada para terceira idade e atividades recreativas, alongamento, Pilates solo e bola, Jump, natação, hidroginástica, ginástica rítmica, tênis de mesa e de quadra, futsal, futebol, handebol, voleibol, basquete, karatê, taekwondo, kung fu, atletismo, xadrez, street dance, capoeira, bocha, vôlei câmbio. Já as atividades desenvolvidas nas ATIs não são todas supervisionadas por profissionais, sendo realizadas individualmente, sendo que os exercícios são compostos por alongamentos de membros inferiores e superiores e a utilização dos aparelhos biomecanicamente projetados para as ATIs e são sempre os mesmos, ou seja, sem estrutura específica se comparados ao grupo G1 (OLIVEIRA; BERTOLINI; MARTINS JÚNIOR, 2014).

Outras modalidades como o método pilates foram estudadas por Tozim et al. (2014) para verificar a sua influência na qualidade de vida, flexibilidade e dor em idosos por meio de um grupo controle e um teste. Os achados do estudo foram que a diminuição do quadro álgico, o aumento da flexibilidade e da qualidade de vida foram proporcionados por meio da prática de exercícios de pilates quando comparada a idosos não praticantes.

$\mathrm{OH}$ et al. (2015) com intuito de identificar os efeitos dos exercícios na água e no solo sobre as funções físicas e a qualidade de vida em idosos da comunidade com risco de quedas, realizaram um estudo intervencional com dois grupos, sendo um de exercícios na água e outro no solo. Os resultados indicaram que os exercícios na água são benéficos para melhorar a qualidade de vida, bem como as atividades físicas de idosos, em comparação com exercício no solo nos idosos da comunidade.

Machado, Santini e Reis Filho (2013) avaliaram e compararam a qualidade de vida de idosas que realizam atividade física habitual com as que praticam treinamento de força. Por meio de questionário WHOQOL-bref apresentaram significância melhores escores nos domínios físicos e ambientais das idosas que praticavam treinamento resistido quando comparada com atividade física habitual. Assim, o treinamento resistido promove uma melhor percepção de qualidade de vida e saúde nos idosos.

Com o objetivo de avaliar os níveis de flexibilidade, autonomina funcional e qualidade de vida em idosos praticantes de yoga, Gonçalves et al. (2011) realizaram um estudo com dois grupos, um praticante de yoga e um controle. Os resultados apontaram que o grupo 
praticante de yoga apresenta melhores resultados na amplitude de movimento articular de ombro, coluna, quadril e joelho, bem como na autonomia e qualidade de vida. Assim, a prática de yoga também contribui para melhora da qualidade de vida, quando comparada com o presente estudo.

Verificou-se diferença significativa (Tabela 5) nos domínios de Relações Sociais $(\mathrm{p}=0,02)$ e Autoavaliação $(\mathrm{p}=0,01)$, indicando que as idosas do G1 possuem maior percepção de avaliação da qualidade em relação a esses aspectos em comparação às idosas do $\mathrm{G} 2$, fato que se dá devido às atividades nos centros esportivos serem realizadas em grupos, gerando uma maior amizade e intimidade, melhorando a sociabilidade e o relacionamento entre tais idosas. Sendo assim, Oliveira, Bertolini e Benedeti (2012) citam que os exercícios realizados em grupos permitem uma otimização das relações sociais e afetivas, proporcionando uma maior satisfação com a vida, aumentando assim a autoeficácia, e consequentemente a avaliação da qualidade de vida do idoso. Em relação a esse domínio, o instrumento WHOQOL-Bref questiona a respeito das relações pessoais das idosas, do apoio recebido por amigos, colegas, parentes e conhecidos, e da atividade sexual. No que diz respeito ao domínio autoavaliação questiona-se como essas idosas avaliam sua avaliação da qualidade e quão satisfeitas estão com a saúde. Sendo assim supõe-se que os exercícios físicos praticados nos Centros Esportivos sejam, então, fatores para melhora desses aspectos.
De acordo com Shimada et al. (2016), a prática de exercício físico em grupo melhora não só as funções físicas e cognitivas, bem como auxilia no envelhecimento saudável. Além disso, ele fornece uma oportunidade para interação social que promove um efeito positivo na qualidade de vida e atividade social.

Segundo Casagrande, Farias e Carpes (2013), os idosos que praticam atividades físicas em grupos de convivência, apresentam menores incidências de déficit cognitivo e de depressão, o que pode estar relacionado com a participação desses idosos em grupos, contribuindo na formação de relação social, assim, consequentemente, promovendo qualidade de vida.

Para Dias et al. (2014) os idosos que praticam exercícios físicos, nas funções cognitivas possuem maiores desempenhos de tempo de reação, de escolha e atenção assistida, quando comparados aos não praticantes.

Silva et al. (2012) realizaram um estudo com objetivo de comparar os níveis de atividade física e qualidade de vida entre os idosos sedentários e os que praticam exercícios físicos regulares. Ao concluir o estudo, os idosos fisicamente ativos apresentaram níveis elevados de atividade física e qualidade de vida, quando comparados ao grupo de sedentários.

Em relação às facetas do WHOQOL-Old, houve diferença significativa apenas na faceta de Intimidade $(\mathrm{p}=0,01)$ evidenciando, também, uma melhor percepção para as idosas do G1, ou seja, as idosas do G1 apresentaram resultados 
positivos em relação à Intimidade, evidenciando que essas têm um sentimento de companheirismo em suas vidas, possuem amor, além de amar e serem amadas, de acordo com os resultados apontados nos questionamentos desse instrumento.

Mesmo com os conhecimentos dos benefícios dos exercícios já estabelecidos para a melhora da saúde, ainda permanecem na população brasileira altas taxas de sedentarismo em todas as faixas etárias. Dessa forma, faz-se necessário incentivar campanhas que promovem mudanças de estilo de vida para melhora da qualidade de vida, sendo intermediada pelo exercício físico (ZAGO, 2010).

Em Pequim, na China, surgiu a ideia de criar as ATIs para que a população realizasse a prática de atividades físicas nas praças públicas ao ar livre (MARANHO, 2013). No ano de 2006, foi implantada a primeira ATI e, atualmente, são 58, por meio de parceria entre as secretarias de saúde, esportes e lazer, além de empresas privadas (SELSON; OLIVEIRA, 2015). Nesse sentido, as ATIs foram implantadas com o intuito da mudança de paradigma na qualidade de vida por meio da atividade física e, assim, favorecendo a saúde nos diferentes níveis de atenção (RADICCHI et al., 2015).

Após implantar as ATIs em Aracajú - SE, observaram-se diversos impactos positivos nos idosos que aderiram à prática de atividade física, tais como, melhoria da aptidão física, do controle e da redução de níveis de pressão arterial, colesterol e glicemia, bem como a melhora da percepção subjetiva de saúde, a qualidade de vida e o convívio social (MENDONÇA; TOSCANO; OLIVEIRA, 2009). A criação de ambientes favoráveis à saúde e a criação de políticas públicas e de ações comunitária são necessários para ampliar a promoção da saúde dos idosos no Brasil (COELHO; VERDI, 2015).

O estudo apresenta algumas limitações como a escassez de estudos com idosos em centros esportivos e ATIs, para fundamentação e discussão dos resultados e a ausência de um grupo controle, composto de idosas não praticantes de exercícios físicos.

Entretanto, mesmo com todas as limitações apontadas, acredita-se que esta pesquisa poderá fornecer aos profissionais da área da Educação Física e da saúde relevantes conhecimentos sobre a importância de se incentivar a prática de exercícios físicos, como um dos componentes fundamentais e determinantes da promoção da saúde do idoso.

\section{Conclusão}

Conclui-se que as idosas praticantes de exercícios físicos nos centros esportivos possuem melhor percepção da qualidade de vida se comparadas às idosas praticantes de exercícios físicos nas ATIs, demonstrando, supostamente, a importância da atividade estruturada, em grupo e com a orientação de um profissional de educação física para a qualidade de vida de idosas. 


\section{Comparative analysis of qualitf of line in elderly practitioners of physical exercise in sports centres and the third age gyms}

\section{Abstract}

This study aimed to analyze the quality of life of elderly women engaged in physical exercise in two separate locations, in sports centers and The Third Age Gyms (TAGs) the city of Maringa - PR, looking specifically compare the quality of elderly living in these places. 70 elderly were assessed, 35 practitioners of physical exercise in sports centers (G1) and 35 in the TAGs (G2). Quality of life was assessed by the WHOQOL -Bref and WHOQOL -Old. Older G1 reached 82.00 score on WHOQOL -Bref, and G2 $77.67(p=0.04)$. In the WHOQOL -Old, G1 had scores of 103 and 94 G2 ( $p=$ 0.01 ), indicating that elderly practitioners of physical exercise in sports centers, have a higher perception of quality of life compared to older practitioners in ITAs

Keywords: Aging. Physical activity. Health promotion.

\section{Referências}

ALENCAR, N. A. et al. Nível de atividade física, autonomia funcional e qualidade de vida em idosas ativas e sedentárias. Fisioterapia e Movimento, Curitiba, v. 23, n. 3, p. 473-481, 2010.

ANDRADE, A.; MARTINS, R. Funcionalidade familiar e qualidade de vida dos idosos. Millenium, Portugal, n. 40, p. 185-199, 2011.

BOETTGE, J. R. et al. Vivências no núcleo universitário da terceira idade: dialogando as percepções da prática. Revista Didática Sistêmica, Rio Grande, v. 17, n. 1, p. 295-297, 2015.
CASAGRANDE, G.; FARIAS, M.; CARPES, P. B. M. Qualidade de vida e incidência de depressão em idosas que frequentam grupos de terceira idade. Revista Brasileira de Ciências do Envelhecimento Humano, Passo Fundo, v. 10, n. 1, p. 52-65, 2013.

CHACHAMOVICH, E.; FLECK, M. P. Desenvolvimento do WHOQOL-BREF. In: FLECK, M. P. A. A avaliação de qualidade de vida: guia para profissionais da saúde. Porto Alegre, RS: Artmed, 2008. 228p.

COELHO, C. S.; VERDI, M. I. M. Políticas e programas de atividade física: uma crítica à luz da promoção da saúde. Health \& Social Change, Florianópolis, v. 6, n. 3, p. 96-108, 2015.

CUNHA, M. F. et al. A influência da fisioterapia na prevenção de quedas em idosos na comunidade: estudo comparativo. Motriz, Rio Claro, v. 15, n. 3, p. 527-536, 2009.

DIAS, R. G. et al. Diferenças nos aspectos cognitivos entre idosos praticantes e não praticantes de exercício físico. Jornal Brasileiro de Psiquiatria, Rio de Janeiro, v. 63, n. 4, p. 326-331, 2014.

ESTEVES, J. V. D. C. et al. Estilo de vida de praticantes de atividades físicas em academias da terceira idade de Maringá-PR. Conexões: Revista da Faculdade de Educação Física da Unicamp, Campinas, v. 8, n. 1, p. 119-129, jan./abr. 2010.

FAUSTINO, A. et al. O perfil comum dos programas de atividade física das academias séniores. Revista De Ciencias Del Deporte, Buenos Aires, v. 2, n. 11, p.135-136, 2015.

FLECK, M. P. A. et al. Aplicação da versão em português do instrumento de avaliação da qualidade de vida "WHOQOL-BREF". Revista Saúde Pública, São Paulo, v. 34, n. 2, p. 178-183, 2000.

FREITAS, C. V. et al. Evaluation of frailty, functional capacity and quality of life of the elderly in geriatric outpatient clinic of a university hospital. Revista Brasileira de Geriatria e Gerontologia, Rio de Janeiro, v. 19, n. 1, p. 119-128, 2016. 
GONÇALVES, L. C. et al. Flexibility, functional autonomy and quality of life (QoL) in elderly yoga practitioners. Archives of Gerontology and Geriatrics, Amsterdam, v. 53, n. 2, p. 158-162, 2011.

INSTITUTO BRASILEIRO DE GEOGRAFIA E ESTATÍSTICA. Senso 2000. Rio de Janeiro, 2000. Disponível em: <http://www. ibge.gov.br>. Acesso em: 2 set. 2015.

LEAL, S. M. O. et al. Efeitos do treinamento funcional na autonomia funcional, equilíbrio e qualidade de vida de idosas. Revista Brasileira Ciência e Movimento, São Paulo, v. 17, n. 3, p. 61-69, 2009.

LIMA, A. C. et al. Benefícios da atividade física para a aptidão do idoso no sistema muscular, na diminuição de doenças crônicas e na saúde mental. Boletim Informativo Unimotrisaude em Sociogerontologia, Amazonas, v. 7, n. 2, p. 34-46, 2016.

MACHADO, E. R.; SANTINI, E.; REIS FILHO, A. Percepção da qualidade de vida por mulheres praticantes de treinamento de força versus praticantes de atividade física habitual. Revista Brasileira de Ciências do Envelhecimento Humano, Passo Fundo, v. 10, n. 2, p. 161-169, 2013.

MARANHO, M. C. A qualidade de vida nos ambientes urbanos: parques e academias ao ar livre no município de Curitiba. Ciência e Cultura, Curitiba, n. 46, p. 45-61, 2013.

MENDONÇA, B. C. A; TOSCANO, J. J. O.; OLIVEIRA, A. C. C. Do diagnóstico à ação: experiências em promoção da atividade física programa academia da cidade aracaju: promovendo saúde por meio da atividade física. Revista Brasileira de Atividade Física \& Saúde, Londrina, v. 14, n. 3, p. 211-216, 2009.

MOREIRA, R. M.; TEIXEIRA, R. M.; NOVA$\mathrm{ES}, \mathrm{K}$. O. Contribuições da atividade física na promoção da saúde, autonomia e independência de idosos. Kairós Gerontologia, São Paulo, v. 17, n. 1, p. 201-217, 2014.
NUNES, B. C. et al. Musculação para terceira idade: experiências e aprendizados. Revista Didática Sistêmica, Rio Grande, v. 17, n. 1, p. 344-347, 2015.

$\mathrm{OH}, \mathrm{S}$. et al. Comparison of the effects of water-and land-based exercises on the physical function and quality of life in communitydwelling elderly people with history of falling: a single-blind, randomized controlled trial. Archives of Gerontology and Geriatrics, Amsterdam, v. 60, n. 2, p. 288-293, 2015.

OLIVEIRA, D. V.; BERTOLINI, S. M. M. G.; MARTINS JÚNIOR, J. Qualidade de vida de idosas praticantes de diferentes modalidades de exercício físico. ConScientiae Saúde, São Paulo, v. 13, n. 2, p. 187-195, 2014.

OLIVEIRA, D. V.; BERTOLINI, S. M. M. G.; BENEDETI, M. R. Avaliação da qualidade de vida de idosos fisicamente ativos por meio do questionário WHOQOL-BREF. Revista Saúde e Pesquisa, Maringá, v. 5, n. 3, p. 547-554, 2012.

OZTURK, A. et al. The relationship between physical, functional capacity and quality of life (QoL) among elderly people with a chronic disease. Archives of Gerontology and Geriatrics, Amsterdam, v. 53, n. 3, p. 278-283, 2011.

PADOIN, P. G. et al. Análise comparativa entre idosos praticantes de exercício físico e sedentários quanto ao risco de quedas. Mundo Saúde, São Paulo, v. 34, n. 2, p. 158-64, 2010.

PALÁCIOS, A. R. O. P.; NARDI, A. C. F. Figueiredo. Academia da terceira idade: promoção da saúde e atividade física em Maringá. Divulg. Saúde Debate, Rio de Janeiro, n. 40, p. 71-76, 2007.

PEDROSO, B.; PILLATI, L. A.; GUTIERREZ, G. L. Cálculos do escore e estatística descritiva do WHOQOL-OLD pelo Microsoft Excel. Revista Brasileira de Qualidade de Vida, Ponta Grossa, v. 4, n. 4, p. 214-219, 2010. 
RADICCHI, M. et al. Descrição dos espaços esportivos de lazer e educação na cidade de Parintins, Amazonas. Revista Brasileira de Atividade Física \& Saúde, Londrina, v. 20, n. 6, p. 626-637, 2015.

SÁ, G. B. A. R. et al. O Programa Academia da Saúde como estratégia de promoção da saúde e modos de vida saudáveis: cenário nacional de implementação. Ciência \& Saúde Coletiva, Rio de Janeiro, v. 21, n. 6, p. 1849-1860, 2016.

SANCHEZ, M. A.; BRASIL, J. M. M.; FERREIRA, I. A. M. Benefícios de um programa de atividade física para a melhoria da qualidade de vida de idosos no estado do Rio de Janeiro. Revista Brasileira de Ciências do Envelhecimento Humano, Passo Fundo, v. 11, n. 3, p. 209-218, 2014.

SANTOS, A. et al. Representações sociais dos usuários do Sistema Único de Saúde do Brasil acerca das práticas corporais/atividades físicas na atenção primária à saúde. CIAIQ, Portugal, v. 2, p. 1436-1445, 2016.

SELSON, G.; OLIVEIRA, R. C. S. Academia da terceira idade como espaço de sociabilidade em Maringá. Revista Científica SMG, Maringá, v. 3, n. 2, p. 4-16, 2015.

SHIMADA, H. et al. Impact of cognitive frailty on daily activities in older persons. The Journal of Nutrition, Health \& Aging, New York, v. 20, n. 7, p. 729-735, 2016.

SILVA, M. F. et al. Relação entre os níveis de atividade física e qualidade de vida de idosos sedentários e fisicamente ativos. Revista Brasileira de Geriatria e Gerontologia, Rio de Janeiro, v. 15, n. 4, p. 635-642, 2012.

TOZIM, B. M. et al. Efeito do método Pilates na flexibilidade, qualidade de vida e nível de dor em idosos. ConScientiae Saúde, São Paulo, v. 13, n. 4, p. 563-570, 2014.

ZAGO, A. S. Exercício físico e o processo saúde-doença no envelhecimento. Revista Brasileira de Geriatria e Gerontologia, Rio de Janeiro, v. 13, n. 1, p. 153-158, 2010. 